\title{
Correlation between sfar score and nasal cytology in paediatric allergic rhinitis patients visiting OPD in Goa Medical College
}

\author{
Joshi $\mathbf{V}^{1}$, Rakesh $\mathbf{R}^{2}$, Silveira $\mathbf{M}^{3}$ \\ ${ }^{1}$ Dr Vaishali M. Joshi, Associate Professor, Department of Pediatrics, Goa Medical College, Goa, ${ }^{2}$ Dr Rakesh R. Senior \\ Resident, Department of Pediatrics, Goa Medical College, Goa, ${ }^{3}$ Dr Silveira Mimi, Professor and HOD, Department of \\ Pediatrics, Goa Medical College, Goa, India.
}

Address for Correspondence: Dr Joshi Vaishali M, Associate Professor, Department of Pediatrics, Faculty Block, Goa Medical College, Bambolim, Goa. E-mail: vaishalijoshi35@rediff.com

\begin{abstract}
Background: The prevalence of allergic rhinitis is $10-20 \%$ worldwide. Allergic rhinitis being a non fatal condition is often trivialized but it causes significant morbidity. The diagnosis of allergic rhinitis is mainly clinical, which often fails in differentiating allergic versus non allergic rhinitis. The importance of this study is to find the effectiveness of nasal mucosal cytology as a diagnostic method for allergic rhinitis, nasal cytology being a simple, non invasive and easily available test may be used as a tool in the diagnosis of allergic rhinitis. Objective: To find the correlation between SFAR score and nasal mucosal eosinophilia in diagnosing allergic rhinitis. Materials and Methods: This was a descriptive prospective hospital based study conducted over a period of two years in Goa medical college. Children in the age group of 1-12 years in whom a clinical diagnosis of allergic rhinitis was made based on ARIA guidelines were included in the study. A subjective definition of allergic rhinitis was made using SFAR score. Nasal smears were collected and examined for eosinophils. A semi quantitative scale was used for grading nasal mucosal eosinophilia. Results: A positive correlation was obtained between nasal smear eosinophilia and SFAR score. The spearman's correlation was 0.426 suggesting a correlation. Conclusion: Nasal mucosal smear eosinophilia can be used as a simple, cheap and non invasive method in diagnosing allergic rhinitis especially in resource limited settings.
\end{abstract}

Key words: Allergic rhinitis, nasal eosinophilia, SFAR score

\section{Introduction}

Allergic Rhinitis, commonly called as hay fever is an IgE mediated hypersensitivity. It is the most common chronic disease suffered by human beings. The prevalence of allergic rhinitis is $10-20 \%$ worldwide and $10-15 \%$ in India [1]. The prevalence of allergic rhinitis has increased during the last two - three decades and it continues to increase.

Allergic rhinitis being a non-fatal condition has been trivialized but it causes significant morbidity to the sufferers. In infancy it may contribute to feeding difficulties and during childhood it may contribute to sleep disturbances, lack in day time concentration, lack of productivity in school, work or sport and social ostracism, thus significantly affecting the quality of

Manuscript received: $8^{\text {th }}$ November 2016

Reviewed: $17^{\text {th }}$ November 2016

Author Corrected: $26^{\text {th }}$ November 2016

Accepted for Publication: $7^{\text {th }}$ December 2016 life $[2,3,4]$. The financial burden to the society due to allergic rhinitis is substantial. Allergic Rhinitis leads to complications of sinusitis and otitis media with effusion [5]. Appropriate management of allergic rhinitis may be an important component in effective management of coexisting or complicating respiratory conditions such as bronchial asthma, sinusitis or sleep apnea. [6,7]. Allergic Rhinitis precedes asthma in the 'allergic march' which begins as infantile eczema proceeds to allergic rhinitis and ends up in bronchial asthma.

There are very few Indian studies on allergic rhinitis, especially in children. There is no published study on allergic rhinitis in Goa, thus this study on allergic rhinitis aims at defining the demographic and clinical pattern of allergic rhinitis in the state of Goa.

The diagnosis of allergic rhinitis is mainly clinical, which often fails in differentiating allergic versus non 
allergic rhinitis. The non availability and cost factors involved in the immunological tests leaves us with no available methods in diagnosing allergic rhinitis. The importance of this study is to find the effectiveness of nasal mucosal cytology as a diagnosing method for allergic rhinitis, nasal cytology being a simple, non invasive and easily available test may be used as a tool in the diagnosis of allergic rhinitis and thus will aid in timely therapeutic intervention $[8,9]$.

\section{Materials and Methods}

This was a descriptive prospective hospital based study conducted in the Department of Paediatrics, Goa medical college, Bambolim over a period of 2 years from September 1, 2012-August 31, 2014, over two allergic seasons.

Children in the age group of 1-12 years visiting paediatric out patient department were included in the study. Children were included in the study in whom a clinical diagnosis of allergic rhinitis was made as per the ARIA guidelines [10], which defines allergic rhinitis as presence of 2 or more recurrent symptoms of excessive sneezing, watery nasal discharge, nasal congestion, nasal itching, red itchy eyes for at least one hour on most of the days or on most days of the season if symptoms are seasonal. Those with fever, purulent nasal discharge and on nasal steroids were excluded from the study

\section{Inclusion Criteria}

- 1-12 Years.

- 2 or more symptoms defining allergic rhinitis i.e recurrent sneezing, watery nasal discharge, nasal congestion, nasal itching, red itchy eyes ,for at least one hour on most of the days or on most days of the season if symptoms are seasonal.

\section{Exclusion Criteria}

- Age less than 1 year and more than 12 years.

- Presence of fever, purulent nasal discharge.
- Patients on nasal steroids.

- Parents not giving consent for nasal swabbing.

Ethical clearance was obtained before the study. A written consent was taken from parents before including in the study. Information was collected using a proforma. A subjective definition of allergic rhinitis was made using the internationally accepted SFAR score [11], a score of more than 7 defines allergic rhinitis. Nasal smears were collected from all children for demonstrating nasal eosinophilia.

\section{Collection and preparation of nasal smear}

After explaining the procedure nasal smears were taken from either nostrils using a cotton swab from the lateral nasal wall from a depth of $1.5-2 \mathrm{~cm}$, rotating the nasal swab thrice, 2 smears were prepared of each patient, smears were dried and stained with wrights stain. Stained smears were examined under high power of a microscope. The number of eosinophils were counted per field.

\section{Semi quantitative scale for nasal cytology}

$$
\begin{array}{ll}
\text { Normal }(+1) & \rightarrow \text { Less than } 10 \text { cells } / \mathrm{hpf} \\
\text { Mild }(2+) & \rightarrow 10-30 \text { cells/hpf or small clumps. }
\end{array}
$$

Moderate $(3+) \rightarrow$ numerous cells or large clumps not covering the entire microscopic field.

Marked $(4+) \rightarrow$ numerous cells or large clumps covering the entire microscopic field.

The grading of nasal smear was done according to Abhey, Rakesh et al, and Shioda and Mahima. [12][13][14]

Stained smears were examined by pathologist under high power of the microscope and smears were graded as per the semi quantitative scale.

Analysis- A computerized analysis was done using SPSS Program.

\section{Results}

In this study majority of the cases were females (57.4\%). Out of 223 cases majority were in the age group of 8-12 years $(58.3 \%)$. Most of the cases were from North Goa district of Goa (57.8\%), Recurrent sneezing was the most common symptom present in $95 \%$ of the patients, $92.35 \%$ had running nose, $60 \%$ had blocked nose and $47 \%$ had nasal itching. Allergic conjunctivitis was present in $31.3 \%$ of the patients..

Cold weather was the most common triggering factor (66.4\%), followed by dust allergy present in $60.5 \%$, allergy to pets in $32.2 \%, 26 \%$ had allergy to strong odours, $21 \%$ had allergy to smoke and food allergy in $4.9 \%$. Family history of atopy was present in $52.4 \%$. 
Table 1: Nasal smear finding in SFAR positive and SFAR negative patients.

\begin{tabular}{|c|c|c|c|c|c|c|}
\hline & & Normal & Mild & Moderate & Severe & Total \\
\hline \multirow{2}{*}{$\begin{array}{c}\text { SFAR } \\
\text { negative }\end{array}$} & count & 62 & 5 & 0 & 0 & 67 \\
\cline { 2 - 7 } & $\%$ & $92.5 \%$ & $7.5 \%$ & $0.0 \%$ & $0.0 \%$ & $100.0 \%$ \\
\hline \multirow{2}{*}{$\begin{array}{c}\text { SFAR } \\
\text { positive }\end{array}$} & count & 73 & 62 & 20 & 1 & 156 \\
\cline { 2 - 7 } & $\%$ & $46.8 \%$ & $39.7 \%$ & $12.8 \%$ & $0.6 \%$ & $100.0 \%$ \\
\cline { 2 - 7 } & count & 135 & 67 & 20 & 1 & 223 \\
\hline Total & $\%$ & $60.5 \%$ & $30.0 \%$ & $9.0 \%$ & $0.4 \%$ & $100.0 \%$ \\
\hline
\end{tabular}

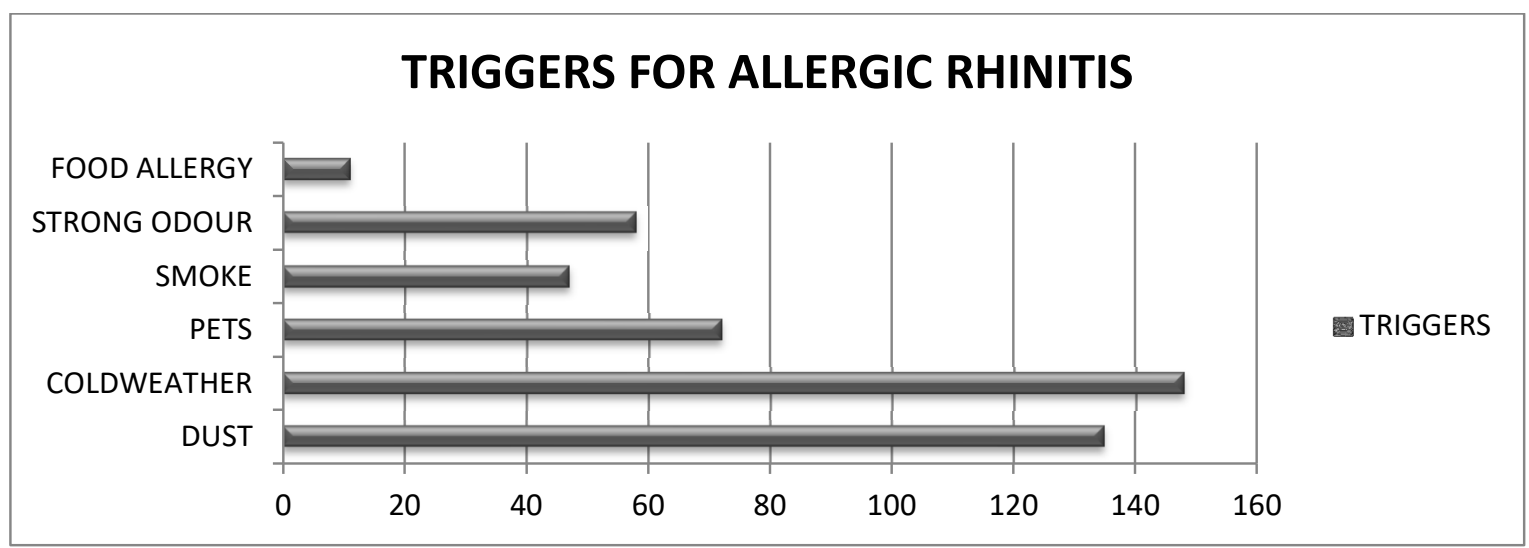

Figure-1 Triggers for Allergic rhinitis:

Cold weather was the most common trigger in $66.36 \%$ patients (148), followed by dust 60.5\% (135), pets $32.2 \%$ (72), strong odors $26 \%$ (58), smoke $21.07 \%$ (47), food allergy $4.9 \%(11)$.

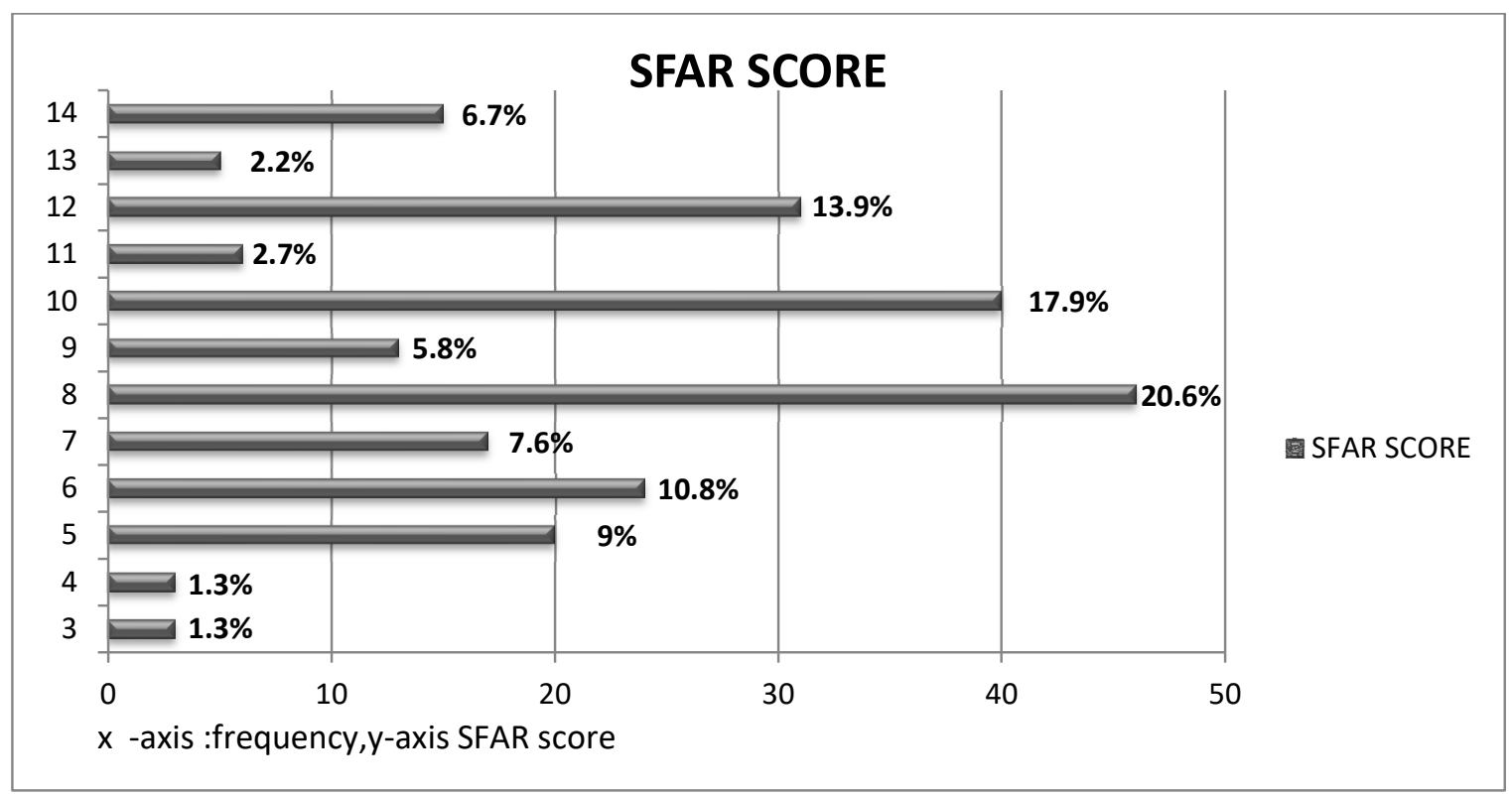

Figure-2: SFAR Score.

SFAR Score of more than 7, which defines allergic rhinitis was present in 70\% (156) of the patients, out of which 97 $(43.4 \%)$ of them had scores of more than or equal to 10 . SFAR score of less than or equal to 7 (no allergic rhinitis as per Sfar) was present in 67 patients $(30 \%)$. 


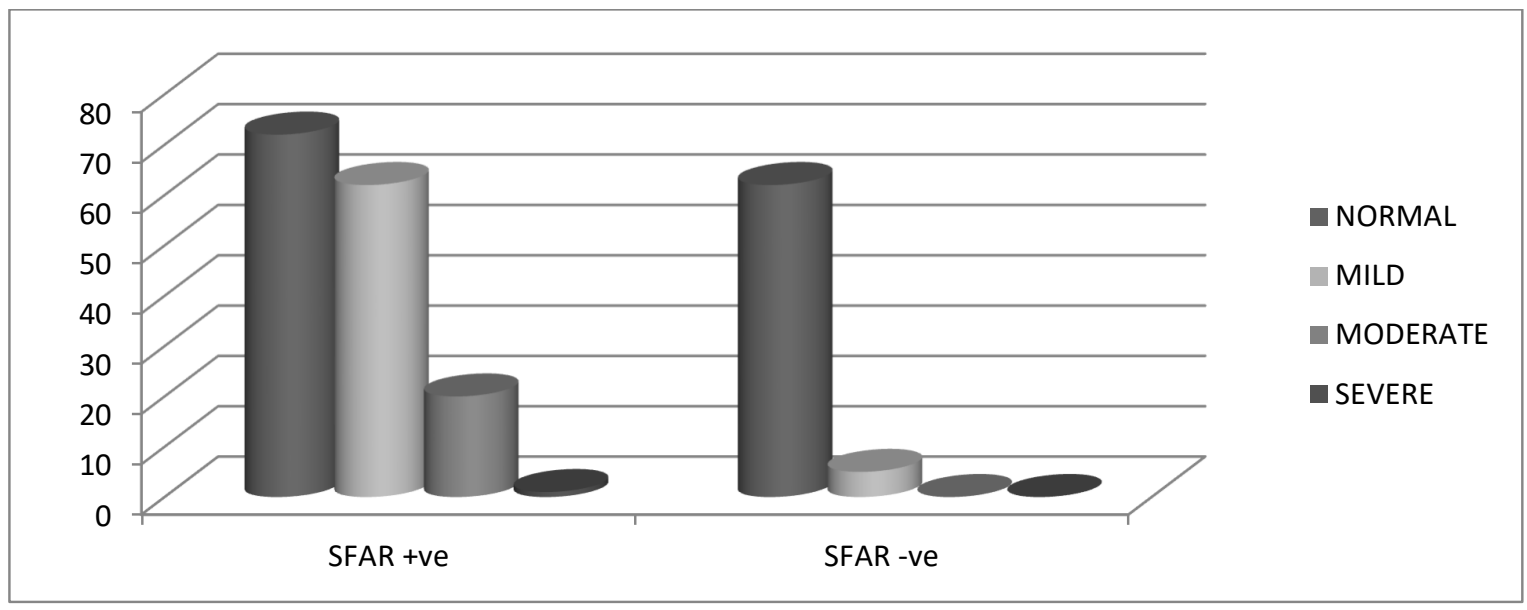

Figure-3: Nasal Smear Eosinophilia:

Nasal smear eosinophilia was normal in $135(60.5 \%)$ of the patients, mild eosinophilia was present in 67 patients (30\%), moderate eosinophilia in 20 (9\%) of them and severe eosinophilia was present in 1 patient $(0.4 \%)$.

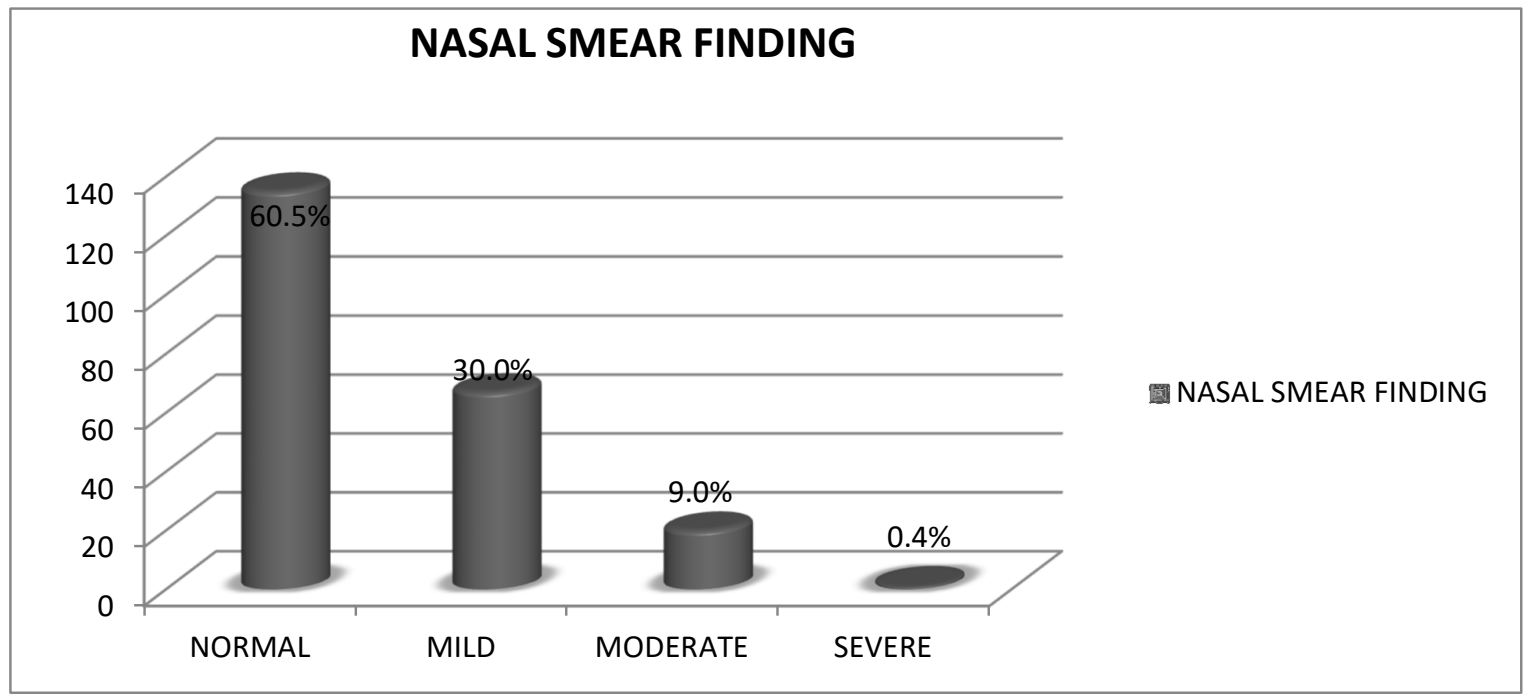

Figure-4: Nasal Smear Finding.

In SFAR positive (score more than 7), group normal smear finding was present in 73, mild eosinophilia in 62, moderate in 20 and severe in 1 among the SFAR negative group normal smear finding was present in 62 and mild eosinophilia in 5 .

Symptoms were seasonal in majority of the cases, $(81.6 \%)$. Otitis media was present in $18.8 \%$ of the patients as a co morbidity followed by eczema in $14.7 \%$, bronchial asthma in $13 \%$, sinusitis in $0.04 \%$.

Nasal mucosal eosinophilia was found in 53\% of the patients with SFAR positive allergic rhinitis. 83 of them had nasal eosinophilia, mild eosinophilia in 36.5\%, moderate eosinophilia in $12.8 \%$ and severe eosinophilia in $0.6 \%$. 89 of them had a positive smear for eosinophilia irrespective of the SFAR score. A positive correlation was obtained between nasal smear eosinophilia and SFAR score. The spearman's correlation was 0.426 suggesting a weak correlation.

Thus it can be concluded that nasal smear eosinophilia can be used as a simple and cheap method in diagnosing allergic rhinitis especially in resource limited setting. 


\section{Discussion}

Allergic rhinitis was predominantly noted among females in our study. A similar finding was seen in the study done by AD Olusesi et al in Nigeria [15].

Recurrent sneezing was the most common symptom in our study affecting $95 \%$ (212 out of 223 ) of the children with allergic rhinitis, followed by running nose affecting $92.3 \%$ (206 out of 223), blocked nose affecting $60 \%$ (134 out of 223) and nasal itching affecting $47 \%$ (105 out of 223). In the study done by S.P.S Yadav et al in Haryana recurrent sneezing was the commonest symptom seen in $100 \%$ of the cases, $90 \%$ had rhinorrhea similar to our study, $72.5 \%$ had nasal obstruction and $57.8 \%$ had nasal itching

Cold weather was the most common allergic trigger as per my study affecting $66.4 \%$ (148) of the children, followed by dust $60.5 \%$ (135), pets $32.2 \%$ (72), strong odours 26\% (58), smoke $21.07 \%(58)$ and food allergy in $4.9 \%(11)$. In the study done by Said et al dust was found as the major trigger for allergy, dust was triggering factor in $39.5 \%$ of the cases, followed by cold weather in $27.9 \%$ cases, perfume in $17.9 \%$ cases and smoke in $3.7 \%$ cases

In our study nasal mucosal eosinophilia was found in $50 \%$ of the patients with SFAR positive allergic rhinitis out of which $36.5 \%$ had mild eosinophilia,12.8\% had moderate eosinophilia and $0.6 \%$ had severe nasal mucosal eosinophia. In the study conducted by $M$ Bakhshaee et al in Iran, nasal mucosal eosinophilia was present in $51 \%$ of the patients. Nasal smear eosinophilia was found in $52.4 \%$ of patients with allergic rhinitis in the study by $\mathrm{N}$ Kumar et al [16]. In the study conducted by $\mathrm{F}$ E Ologe et al mild eosinophilia was not present in any patients with allergic rhinitis, moderate eosinophilia in $71.6 \%$ patients and severe eosinophilia in $28.4 \%$ cases [17].

The study by Chanda et al has shown a lower nasal smear eosinophilia of $40 \%$ in the study they conducted at PGIMS, Rohtak, Haryana [13].

We found that there is a correlation between SFAR score and nasal smear eosinophilia, the spearman correlation coefficient was 0.429 . A correlation of 0.88 between nasal mucosal cytology and SFAR score was obtained by F E Ologe et al. In this study they have found a strong correlation between nasal smear eosinophilia \& SAFAR score [17].
Acknoledgement- This manuscript is extracted from the post graduate thesis done by Dr Rakesh.R, which was successfully completed under the supervision of Dr Vaishali Joshi and Dr Mimi Silveira and with the close cooperation of department of pediatrics and pathology. The smear microscopy was done by pathologist Dr Adhisha. A.S and statistical analysis was done by statistician Pallavi Nachinolkar.

Key Messages: Nasal mucosal cytology for eosinophilia can be used as simple, cheap and noninvasive method for diagnosing allergic rhinitis in settings where complex immunological tests are not available.

Capsule Summary: SFAR is a validated and accepted scoring system for allergic rhinitis however nasal cytology is not validated. A positive correlation of nasal mucosal eosinolphilia implies that nasal eosinophilia will serve as a definite diagnostic test for allergic rhinitis especially in resource limited settings.

Funding: Nil, Conflict of interest: None initiated, Permission from IRB: Yes

\section{References}

1. S.N. Gaur, K. Gupta, S. Rajpal, A.B. Singh, A. Rohatgi. Prevalance of bronchial asthma and allergic rhinitis among school children in Delhi. Indian J Allergy Asthma Immunol 2006; 20(2) : 90 - 97.

2. Bousquet J, Neukirch F, Bousquet PJ et al. Severity and impairment of allergic rhinitis in patients consulting in primarycare. J Allergy Clin Immunol. 2006 Jan; 117(1):158-62. Epub 2005 Dec 2. DOI: 10.1016/j. jaci.2005.09.047.

3. Green RJ, Davis G, Price D. Concerns of patients with allergic rhinitis: the AllergicRhinitis Care Programme in South Africa. Prim Care Respir J. 2007 Oct; 16(5):299-303. DOI: 10.3132/pcrj.2007.00062.

4. Léger D, Annesi-Maesano I, Carat F, Rugina M et al. Allergic rhinitis and its consequences on quality of sleep: an unexplored area. Arch Intern Med. 2006 Sep 18;166 (16): 1744-8.DOI:10.1001/ archinte. 166.16. 1744 .

5. Lucente FE. Rhinitis and nasal obstruction. Otolaryngol Clin North Am. 1989 Apr;22(2):307-18. 
6. Kurukulaaratchy RJ, Matthews S, Arshad SH. Defining childhood atopic phenotypesto investigate the association of atopic sensitization with allergic disease. Allergy. 2005 Oct;60(10):1280-6. DOI: 10.1111/j.13989995.2005.00890.x.

7. G.Roberts, M.Xatzipsalti, L.M.Borrego, A.Custovic, S.Halken, P.W.Hellings, N.GPapadopoulos, G.Rotiroti, G. Scadding, F.Timmermans \& E.Valovirta. Paediatric rhinitis: position paper of the European Academy of Allergy and Clinical Immunology.European Journal of Allergy and Clinical Immunology. July 2003.

8. Rondón C, Romero JJ, López $\mathrm{S}$ et al. Local IgE production and positive nasal provocation test in patients with persistent nonallergic rhinitis. J Allergy Clin Immunol. 2007 Apr;119(4):899-905. Epub 2007 Mar 2. D OI: 10.1016/j.jaci.2007.01.006.

9. Romero JN, Scadding G. Eosinophilia in nasal secretions compared to skin prick test and nasal challenge test in the diagnosis of nasal allergy. Rhinology. 1992 Sep;30(3):169-75.

10. Brozek JL, Bousquet J, Baena-Cagnani CE et al. Allergic Rhinitis and its Impact on Asthma (ARIA) guidelines: 2010 revision. J Allergy Clin Immunol. 2010Sep;126(3):466-76.doi:10.1016/j.jaci.2010.06. 047.

11. Annesi-Maesano I, Didier A, Klossek M, Chanal I et al. The score for allergic rhinitis (SFAR): a simple and valid assessment method in population studies. Allergy. 2002 Feb;57(2):107-14.

12. Sood A. Diagnostic significance of nasal eosinophilia in allergic rhinitis. Indian J Otolaryngol Head Neck Surg. 2005 Jan;57(1):13-6. doi: 10.1007/ BF02907618.

13. Rakesh Chanda, Ajay Kumar Aggarwal, G.S. Kohli, T.S. Jaswal, K.B. Gupta. Compatitive study of nasal smear and biopsy in patients with allergic rhinitis. Indian J Allergy Asthma Immunol 2002; 16(1) : 27-31.

14. Shioda H, Mishima T. Significance of mast cells in nasal smears from patients with food allergy. J Allergy. 1966 Jun;37(6):321-8.

15. AD Olusesi,MA Said,EJ Amodu.A correlation of symptomatology with nasal smear eosinophilia in non infectious chronic rhinitis preliminary report. Nigerian Journal of Clinical Practice ,September 2007 vol 10(3): $238-4$.

16. Naveen Kumar,Kiran Bylappa,Ramesh AC,Swetha Reddy.A study of eosinophil count in nasal and blood smear in allergic respiratory diseases in a rural setup.Internet Journal of Medical Update 2012 January, $7(1): 40-6$.

17. Ologe FE,Adebola SO,Dunmade AD,Adenji KA,Oyejola BA. Symptom score for allergic rhinitis. Otolaryngol Head Neck Surg.2013 Apr,148(4):557-63.

\section{How to cite this article?}

Joshi V, Rakesh R, Silveira M. Correlation between sfar score and nasal cytology in paediatric allergic rhinitis patients visiting OPD in Goa Medical College. J PediatrRes.2016;3(12):906-911.doi:10.17511/ijpr.2016.i12.10. 